\title{
Human Performance in Cooperative Virtual Environments: the Effect of Visual Aids and Oral Communication
}

\author{
Sehat Ullah ${ }^{1}$, Paul Richard ${ }^{2}$, Samir Otmane ${ }^{1}$, Mickael Naud ${ }^{2}$, and Malik Mallem ${ }^{1}$ \\ ${ }^{I}$ IBISC Laboratory, University of Evry, France. \\ ${ }^{2}$ LISA Laboratory, University of Angers, France.
}

\begin{abstract}
The Cooperative virtual environments, where users simultaneously manipulate objects, are one of the subfields of Collaborative virtual environments (CVEs). In this paper we simulate the use of two string based parallel robots in cooperative teleoperation task. Two users setting on separate machines connected through local network operate each robot. In this context, we investigate the effects of visual aids and oral communication on cooperation, co-presence and users performance. Ten volunteers had to cooperatively perform a peg-in-hole task. A second group of ten subjects perform the same task in a single user setup. The objective of the two experiments is twofold, firstly to compare the task's complexity of single user setup with that of the cooperative environment. Secondly we examine the influence of visual aids and oral communication on user's performance in the two different setups. Results revealed that shadow has a significant effect on task execution while arrows and oral communication not only increase users' performance but also enhance the sense of co-presence and awareness. We also observed that cooperative manipulation was more difficult as compared to single user manipulation.
\end{abstract}

Index Terms - CVEs, cooperative teleoperation, human performance, visual aids, oral communication.

\section{INTRODUCTION}

The successful advancements in the field of high quality computer graphics and the capability of inexpensive personal computers to render high-end 3D graphics in a more realistic manner has made virtual reality (VR) feasible to be used in many areas such as industrial design, data visualization, training etc. Similarly, there are other domains of VR application such as medical [26, 22, 26, 11], textile and fashion [16], assembling, repairing and education [5], etc.

Human beings often perform their work (from simple to complex ones) in a collaborative manner, that is why VR scientists initiated the development of virtual environments (VEs) supporting collaborative work. A CVE is a computer generated world that enables people in local/remote locations to interact with synthetic objects and representations of other participants within it. The applications of such environments are in military training, telepresence, collaborative design and

Manuscript received on 12 March 2009

E-Mail: sehatullah@ibisc.univ-evry.fr entertainment. Interaction in CVE may take one of the following forms [21]:

- Asynchronous: It is the sequential manipulation of distinct or the same attributes of an object, for example a person changes an object's position, and then another person paints it. Another example is, if a person moves an object to a place, then another person moves it further.

- Synchronous: It is the concurrent manipulation of distinct or the same attributes of an object, for example a person is holding an object while another person is painting it, or when two or many people lift or displace a heavy object together.

The concurrent manipulation is also termed as Cooperative Manipulation or Cooperative work.

In order to carry out a cooperative task efficiently, the participants need to feel the presence of others and have means of communication with each other. The communication may be verbal or non verbal such as pointing to, looking at or even through gestures or facial expressions. Similarly the participants must have a common protocol for task execution. The design and implementation of a system with these capabilities specially for distant users has really been a challenging job for the researchers. For example the architecture of the virtual world may be client server or a replicated one. In case of client-server architecture the known problems of network load and latency arise.

Similarly in replicated solution the consistency of two or more sites need to be addressed. We implement the VE designed for cooperative work in replicated architecture and seek solution to network load/latency and VE consistency in a unique way. Similarly to feel the presence of others and to make cooperative work easier and more intuitive, we augment the environment with visual aids and oral communication and investigate their effects on user performance in a peg-in-hole task [10, 32].

This section is followed by the related work. Section 3 describes the proposed system and the hardware platform used for the experiments. Section 4 presents the peg-in hole experiment I in single user setup. Section 5 discusses the experiment II in which the same task is cooperatively performed. Section 6 is dedicated to the comparative analysis of experiment I \& II. Section 7 gives conclusion and some tracks for future work. 


\section{RELATED WORK}

A lot of work has already been done in the field of CVE, for example MASSIVE provides a collaborative environment for teleconferencing [12]. Most of this collaborative work is pertinent to the general software sketch, the underlying network architecture [7, 30] and framework [4, 18].

Basdogan et al. have investigated the role of force feedback in cooperative task. They connected two monitors and haptic devices to a single machine [6]. Similarly, sallnas et al. have reported the effect of force feedback over presence, awareness and task performance in a CVE. Similarly, they connected two monitors and haptic devices to a single host [27]. A heterogeneous scalable architecture has been given, which supports haptic interactions in collaborative tasks [29]. Other important works that support the cooperative manipulation of objects in a VE include [15, 14, 19, 3] but all these systems require heavy data exchange between two nodes to keep them consistent.

Visual, auditory and tactile cues have already been used both in single user VR and teleoperation systems as a substitute for haptic feedback $[17,23]$. Sensory substitution may also be used as a redundant cue to avoid the possible force feedback instabilities in presence of small delays.

\section{DESCRIPTION OF THE SYSTEM}

In this section we present a system that enables two users, connected through Local Area Network (LAN), to cooperatively manipulate virtual objects using string-based simulated parallel robots in a VE. Secondly we present how oral communication and the visual aids (shadow and arrows) may assist the cooperative manipulation of objects.

The VE for cooperative manipulation has a simple cubic structure (has side of $36 \mathrm{~cm}$ ), consisting of three walls, floor and a ceiling. Furthermore the VE contains four cylinders each with a distinct color and standing lengthwise in a line (see Fig. 1). In front of each cylinder at the distance of $30 \mathrm{~cm}$ there is a torus of the same color. All cylinders are of the same size $1.5 \mathrm{~cm}$. The red, green, blue and yellow toruses have inner radii of 1.6, 1.8, 2.0 and $2.20 \mathrm{~cm}$ respectively. Cylinders and toruses have $4 \mathrm{~cm}$ distance between them. We have modeled two SPIDAR (3DOF) to be used as robots $[28,24,31]$. At each corners of the cube a motor for one of the SPIDAR has been mounted. The end effectors of the SPIDARs have been represented by two spheres of distinct color. Each end effector uses 4 wires (same in color) for connection with its corresponding motors. Therefore, user's movements are constrained by the wire arrangement of the SPIDAR.

One of the important tasks related to collaborative/ cooperative system is the representation of users in the virtual world. This is normally carried out using avatars [21, 13, 14, 9] or some other representations like virtual hands or balls $[8,5,19$, 20]. We use two spheres which are identical in size but different in colors (one is red and the other is blue) so that the users may feel the presence of others. Each pointer controls the movements of an end effector. Once a pointer collides with its corresponding end effector, the later will follow the movements of the former. In order to lift and/or transport a cylinder the red end effector will always rest on right and blue on left of the cylinder.

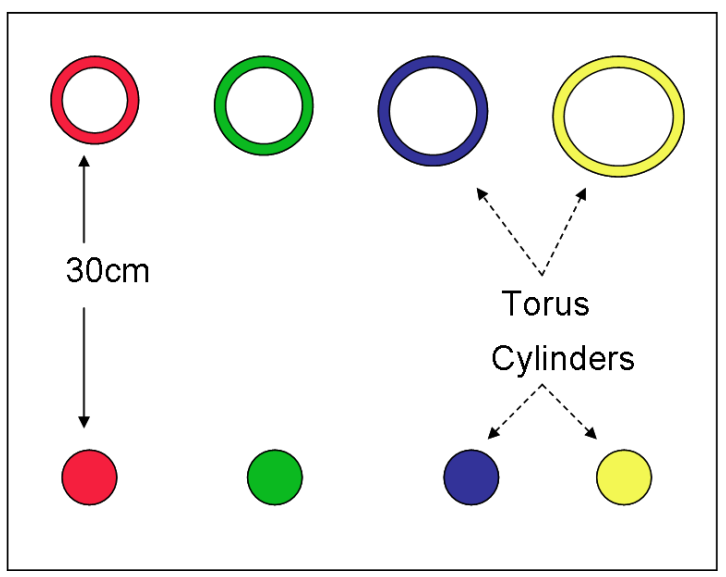

Fig. 1. Illustration of the virtual environment (Top view)

\subsection{Use of visual aid and oral communication in cooperative work}

Cooperative work is really a challenging research area, specially when the users are connected through LAN or WAN, because there are a number points to be treated. For example to sense the presence of others and to have awareness of where is and what is the status of other partners is essential and may have profound effects on cooperation. Similarly the cooperating persons should also have some feedback to know, when they can start together, can leave each other (when task is finished), or if there is some interruption during task. For this purpose we exploit visual feedback and oral communication. In visual channel we make use of arrows and objects' shadows.

If any user moves to touch a cylinder on its proper side, an arrow appears pointing in the opposite direction of the force applied by the end effector on the object (see Fig. 2). The arrow has many advantages, for example it indicates the collision between an end effector and cylinder. Similarly during the transportation, if any user looses control of the cylinder, his/her arrow will disappear and the cylinder will stop moving. The second user will just wait for the first one to come back in contact with the cylinder. It means that the two users will be aware of each other's status via arrows during task accomplishment.

Our current system is a desktop environment and do not support stereoscopic display. In order to have the knowledge of perspective positions of various objects in the VE, we make use of shadow for all objects in the environment. The shadows not only give information about the two end effector's contact with cylinder but also provide feedback about the cylinder's position relative to its corresponding torus during transportation.

Human beings frequently make use of oral communication while performing a collaborative or/and cooperative task. In order to accomplish the cooperative work in a more natural manner, to achieve high performance and increase co-presence and awareness, we make use of oral communication in our system. For this purpose we use TeamSpeak software that 
allows the two users to communicate over the network using a headphone equipped with microphone [1]. The oral communication allows the users to negotiate and inform each other about various events, such as increase or decrease in speed, losing control of the cylinder and arriving over the torus. The following conditions are checked once the two end effectors touch a cylinder (see Fig. 3).

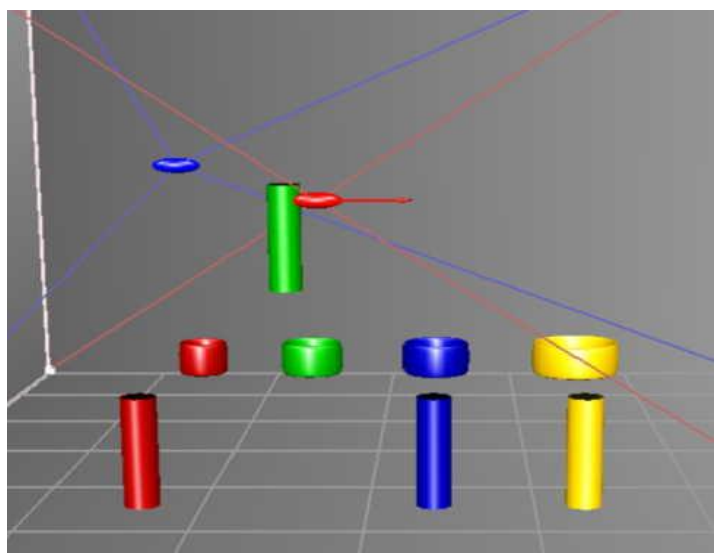

Fig. 2. Illustration of the appearance of arrow

$$
\begin{gathered}
D_{h} \geq 2 R_{c}-K \\
D_{v} \leq T
\end{gathered}
$$

In equation $1, \mathrm{Dh}$ represents the horizontal distance between the centers of the two spheres, $\mathrm{Rc}$ is the radius of the cylinder and $\mathrm{K}$ is a positive constant. This check ensures that the spheres must not completely penetrate the cylinder and should remain visible during the task. In equation 2, Dv represents the vertical distance between the centers of the two spheres that must be less than or equal to a threshold $\mathrm{T}$. When conditions in equation 1 and equation 2 are both satisfied then users can cooperatively move the cylinder.

\subsection{Framework for Cooperative VE}

The framework plays a very important role in the success of collaborative and/or cooperative VEs. It is pertinent to, how different users will have access to the same virtual world and data (i.e centralized, distributed or replicated), what protocol (TCP ,UDP, etc) to be used and what kind of data should flow through network to keep consistency as well [8]. We use a complete replicated approach and install the same copy of the VE on two different machines.

\subsection{Experimental Setup}

We installed the software on two Pentium 4 type personal computers connected through local network. Each machine had processor of 3GHZ and 1GB memory. Each system is equipped with standard graphic and sound cards. Both systems used 24 inch plate LCD tv screen for display.

Similarly each VR station is equipped with a patriot polhemus [2] as input device. The workspace that polhemus support is a half sphere of $50 \mathrm{~cm}$ radius. The software was developed using $\mathrm{C}++$ and OpenGL Library.

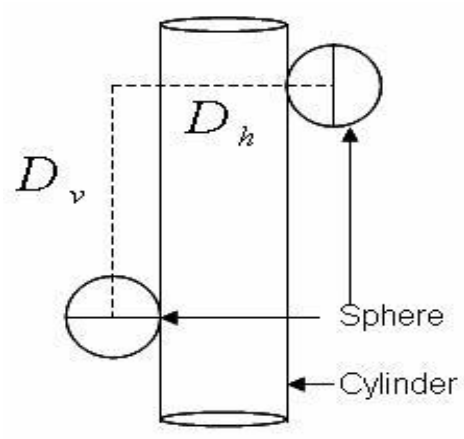

Fig. 3. Illustration of conditions and way of cooperative manipulation

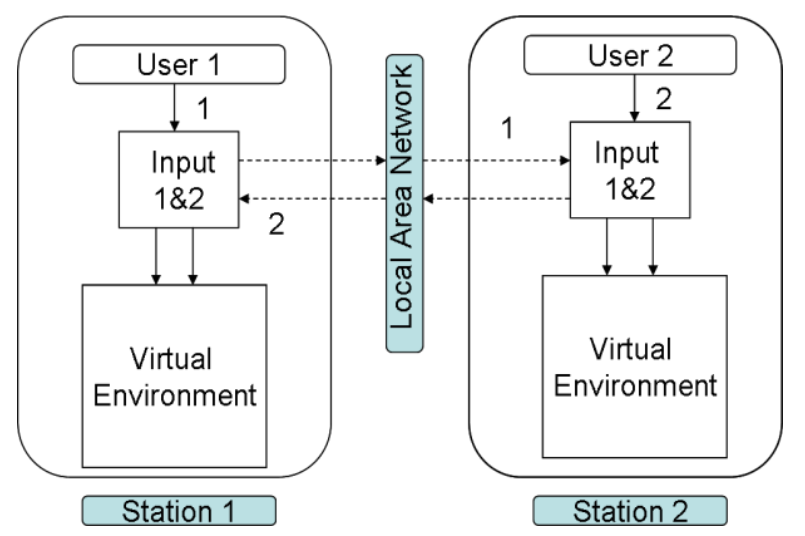

Fig. 4. Illustration of the framework of cooperative virtual environment

\section{EXPERIMENT I}

In this experiment the peg-in-hole task was carried out in a single user setup. For this purpose two polhemus sensors were used on the same machine. The two sensors were attached to the right hand of the users in a way that the sensors corresponding to the red and blue sphere were on the index finger and thumb respectively (see Fig. 5). This experiment was performed by ten volunteers consisting of five male and five female. All of them were master students and were right handed. Each subject was given a pre-trial along with a short briefing. Here the task for the users was to grasp the cylinders via end effectors and put them in its corresponding torus. We tested only conditions $\mathrm{C} 1$ (Shadow), C2 (Shadow + Arrow) and C4 (No aid) in a counter balanced manner. There were four trials under each condition and the order of selection was sequential starting from the red. The evaluation is based on task completion time, errors and user's response collected through a questionnaire.

\subsection{Task completion time}

Fig. 6 illustrates the average task completion time in single user setup for conditions C1 (Shadow), C2 (Shadow +Arrow) and C4 (No aid). For task completion time the $\operatorname{ANOVA}(F(3,9)=7.52$, $\mathrm{p}<0.05)$ is significant. Comparing the task completion time of $\mathrm{C} 1$ and $\mathrm{C} 4$, we have $6.34 \mathrm{sec}$ (std 1.51) and $8.09 \mathrm{sec}$ (std 1.14) 
respectively with a significant ANOVA. It shows that users performed better in condition $\mathrm{C} 1$ as compared to

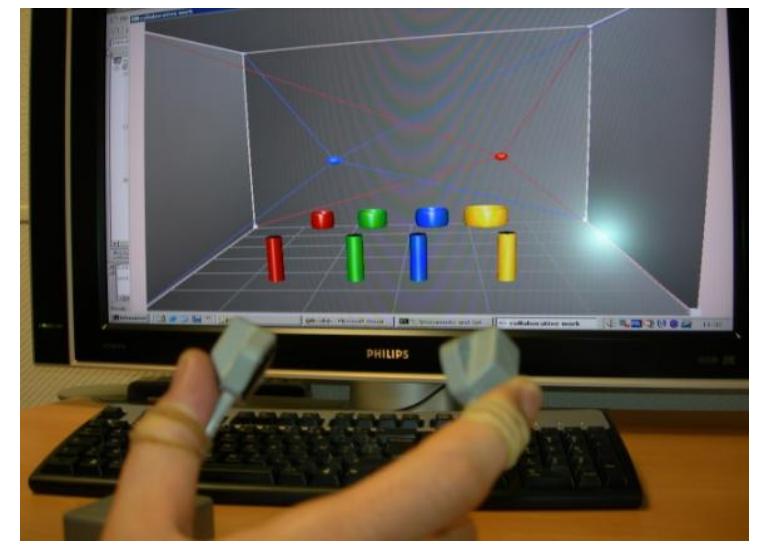

Fig. 5. Illustration of polhemus sensors in single user setup

condition C4. Similarly C2 (6.27 sec, std 0.82) and C4 (8.09 sec, std 1.14) also gives significant ANOVA results. Therefore, users performed better in condition $\mathrm{C} 2$ as compare to condition $\mathrm{C} 4$. On the other hand comparing the task completion time of $\mathrm{C} 1$ and $\mathrm{C} 2$, We have $6.34 \mathrm{sec}$ (std 1.51) and $6.27 \mathrm{sec}$ (std 0.82) respectively with a non significant ANOVA. This result shows that shadow has an influence but arrow has no influence on task performance in the single user setup.

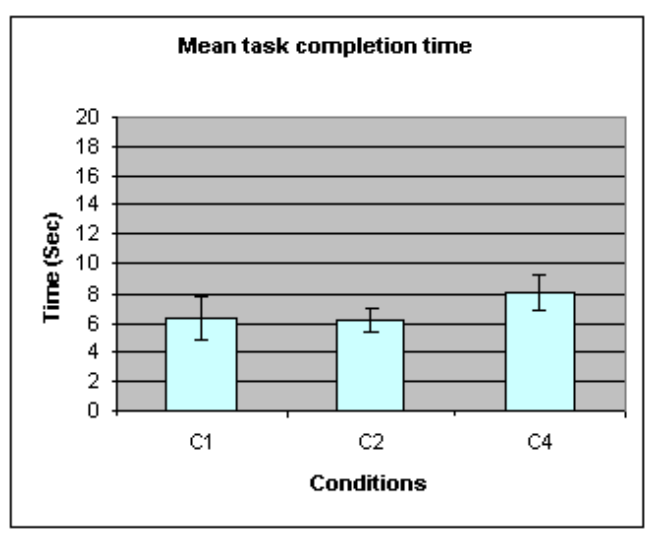

Fig. 6. Task completion time under various conditions in single user setup

\subsection{Errors in task completion}

Fig. 7 illustrates the average error in single user setup for conditions C1 (Shadow), C2 (Shadow +Arrow) and C4 (No aid). The loss of cylinder's control is considered as an error/drop. We recorded the number of errors for each cylinder under each condition. A global error analysis is presented. For errors in task completion, the ANOVA $(\mathrm{F}(3,9)=0.49, \mathrm{p}>0.05)$ is not significant. Here $\mathrm{C} 1$ has mean of 1.16 errors with std 0.72 . Similarly C2 and C4 have 1.17 (std 0.48), 1.47 (std 1.02) means respectively. The user had more control over his/her finger's positions. It resulted in less errors in all conditions.

\subsection{Subjective evaluation}

For subjective evaluation users responded to the questionnaire after task completion. The questionnaire had the following questions.

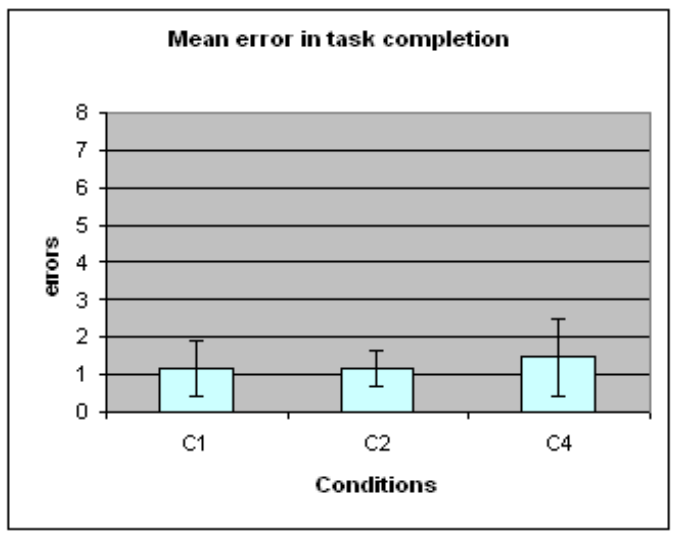

Fig. 7. Illustration of errors for various conditions in single user setup

- What condition did you prefer? (C1, C2, C4 )

Here $90 \%$ user opted for $\mathrm{C} 1$ while $10 \%$ for $\mathrm{C} 2$.

- What feedback helped you more for task accomplishment? ( $\mathrm{C} 1, \mathrm{C} 2, \mathrm{C} 4$ )

Here $80 \%$ users were for $\mathrm{C} 1$ while the $20 \%$ were for $\mathrm{C} 2$.

- Which part of the task was more difficult? (Grasping,

Transportation, Placement )

The response was $20 \%, 70 \%$ and $10 \%$ for grasping, transportation and placement respectively.

\subsection{User learning}

Learning is defined as the improvement of group performance during task repetition. In figure 8 the four trials (T1, T2, T3 and T4) are represented along x-axis. Conditions are differentiated through different colors. The results show that applying condition $\mathrm{C} 1$, the subjects completed the task in 7.1 $\mathrm{sec}(\mathrm{std}=1.32)$ in the first trial and in $5.37 \mathrm{sec}(\mathrm{std}=0.92)$ in the fourth trial. They completed the task under condition $\mathrm{C} 2$ in a mean time of $6.22 \mathrm{sec}(\mathrm{std}=1.07)$ in the first trial, while took $5.77 \mathrm{sec}(\mathrm{std}=0.95)$ in the fourth trial. Similarly we have the mean time of $8.05 \mathrm{sec}(\mathrm{std}=2.02)$ under condition $\mathrm{C} 4$ for the first trial and $6.9 \mathrm{sec}(\mathrm{std}=1.04)$ for the last trial. This results in performance improvement of 24.36, 7.24 and 14.28 percent for conditions $\mathrm{C} 1, \mathrm{C} 2$ and $\mathrm{C} 4$ respectively.

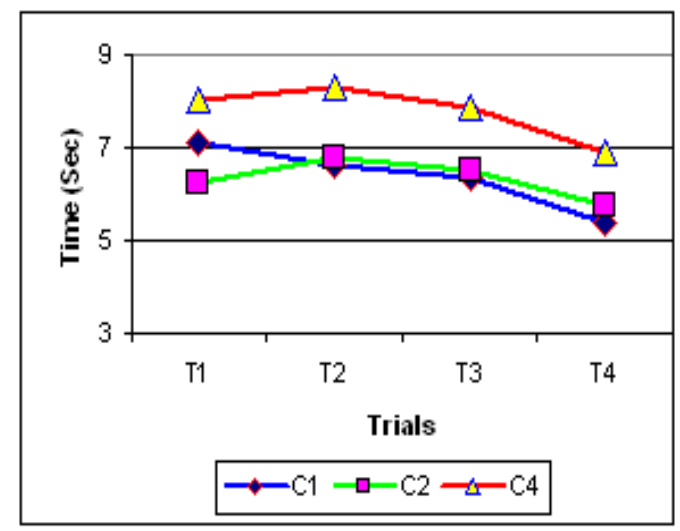

Fig. 8. Illustration of user learning in single user setup for various conditions 


\section{EXPERIMENT II}

The second experiment is pertaining to cooperative manipulation while in the first experiment the same task was performed by users in a single user setup.

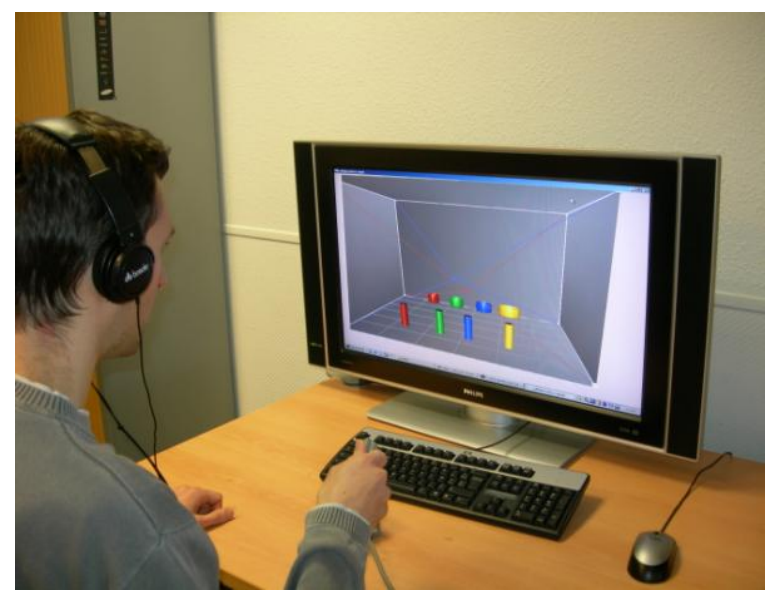

Fig. 9. Illustration of the experimental setup

\subsection{Procedure}

In order to evaluate the system and investigate the effect of visual aids and oral communication on user performance in cooperative object manipulation, we carried out experiment II. For this purpose a new group of ten volunteers consisting of five male and five female participated. They were master and $\mathrm{PhD}$ students and had ages from 22 to 35 . All the participants performed the experiment with same person who was expert of the domain and also of proposed system.

Each subject was given a short briefing about the experiment and to get them familiar with the system. They were also given a pre-trial in which they experienced all feedbacks. The users needed to start the application on their respective machines. After the successful network connection between the two computers the users could see the two spheres (red and blue) as well as the two end effector of SPIDARs on their screens. Seeing the two spheres they were required to bring their polhemus controlled spheres in contact with their respective end effectors (i.e. red + red and blue +blue). The red sphere was assigned to the expert while the subjects were in charge of the blue one. In order to pick up the cylinder the expert needs to touch it from right while the subject should rest on its left. The experiment was carried out under the following four conditions.

- $\mathrm{C} 1=$ only shadow

- $\mathrm{C} 2=$ shadow + arrows

- $\mathrm{C} 3=$ shadow + arrows + oral communication

- $\mathrm{C} 4=$ No aid

All the ten groups performed the experiment using counter balance combinations of the four conditions. We recorded the task completion time for each cylinder. The time counter starts for a cylinder once the two end effectors have an initial contact with it, and stops when it is properly placed in the torus. The indicator for the proper placement of cylinder is the change in color (white) of the torus. Similarly we recorded the number of times the cylinder was dropped as errors. After task completion we gave each user a questionnaire in order to have subjective feedback. Fig. 9 shows a novice user on his station while performing the task.

\subsection{Task}

The experiment for the users was to cooperatively pick up a cylinder and put it into the torus whose color matches with the cylinder. The users were required to place all cylinders in their corresponding toruses in a single trial. Each group performed exactly four trials under each condition. Thus each user had 64 manipulations of cylinders under all conditions. The order of selection of the cylinders was also the same for all groups i.e. to start from the red, go on sequentially and finish at yellow (right).

In following subsections we present and analyze the results of task completion time and also the errors made during task accomplishment. Similarly the user's responses collected through the questionnaire is also thoroughly examined and discussed.

\subsection{Task completion time}

For task completion time the $\operatorname{ANOVA}(\mathrm{F}(3,9)=16.02, \mathrm{p}<$ $0.05)$ is significant. Comparing the task completion time of $\mathrm{C} 1$ and $\mathrm{C} 2$, we have $30.07 \mathrm{sec}$ (std 6.17) and 22.39 sec (std 3.10) respectively with a significant ANOVA. This result shows that arrow has an influence on task performance. Similarly comparing C4 (mean 38.31 sec, std 7.94) with $\mathrm{C} 1$ also gives significant ANOVA. This indicates that only "shadow" as compared to"No aid" also increases user performance. Now we compare the mean $22.39 \mathrm{sec}$ (std 3.10) of C2 with that of C3 (24.48 sec std 3.93), the ANOVA result is not significant. It shows that users had almost the same level of performance under $\mathrm{C} 2$ and $\mathrm{C} 3$. On the other hand the comparison of $\mathrm{C} 2$ and C3 with C4 (mean $38.31 \mathrm{sec}$, std 7.94) both have statistically significant results (see Fig. 10).

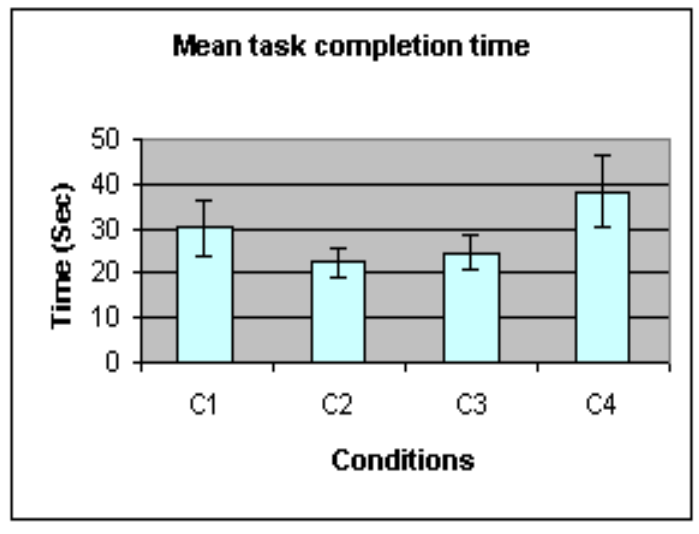

Fig. 10. Task completion time under various conditions 


\subsection{Error in task completion}

When one or both users were detached from the cylinder during task accomplishment, it was considered as an error. We recorded the number of errors for each cylinder under each condition. We present a global error analysis for each condition (see Fig. 11). Here $\mathrm{C} 1$ has mean of 8.6 errors with std 4.6. Similarly C2, C3 and C4 have 6.6 (std 3.5), 6.4 (std 3.2) and 11.7 (std 5.7) means with std respectively. The errors are less in conditions $\mathrm{C} 2$ and $\mathrm{C} 3$ as compared to conditions $\mathrm{C} 1$ and $\mathrm{C} 4$.

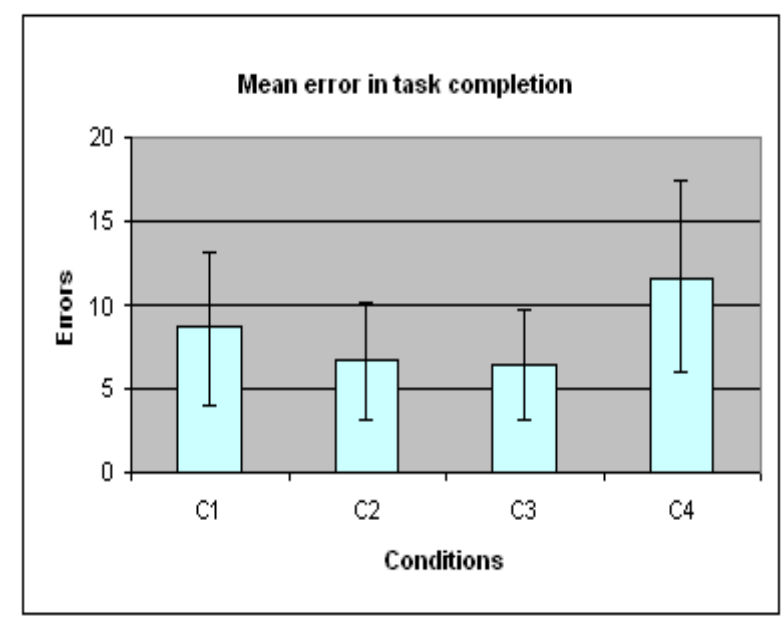

Fig. 11. Illustration of error for various conditions

\subsection{Subjective evaluation}

In this section we analyze the response collected through the questionnaire. The questionnaire had five questions with three to four options for response. For each question the subjects had to select the options in their order of preference.

- Q1: What condition did you prefer? Classify in order of preference.

(a) $\mathrm{C} 1$ (b) $\mathrm{C} 2$ (c) $\mathrm{C} 3$ (d) $\mathrm{C} 4$

For this question $90 \%$ subjects placed $\mathrm{C} 3$ as their first option while $10 \%$ put it on the 2 nd. C2 was marked by $10 \%, 70 \%$ and $20 \%$ subjects as their first, second and third priority respectively. Similarly $\mathrm{C} 1$ got $30 \%$ and $70 \%$ opinion for second and third position respectively. On the other hand $\mathrm{C} 4$ was placed by all users at last position.

- Q2: What feedback helped you more in task accomplishment? Classify in order of preference.

(a) Shadow (b) Arrow (c) Oral communication

For the 2nd question, Shadow was marked by 50\%, 30\% and $20 \%$ users as first, second and third priority respectively. Only $10 \%$ users placed arrow on first position while $40 \%$ and $50 \%$ users placed it on 2nd and 3rd position respectively. Oral communication was prioritized for $2^{\text {nd }}$ position by $40 \%$, while 1 st and 3rd position each got $30 \%$ votes.

- Q3: In which condition you perceived better the actions of your collaborator? Classify in order of preference.
(a) $\mathrm{C} 1$ (b) $\mathrm{C} 2$ (c) $\mathrm{C} 3$ (d) $\mathrm{C} 4$

For this question $90 \%$ subjects placed C3 at first priority while $10 \%$ put it on the 3rd. C2 got $10 \%, 70 \%$ and $20 \%$ opinions for 1st, 2nd and 3rd priority positions respectively. C1 was marked by $30 \%$ and $70 \%$ users for 2 nd and $3^{\text {rd }}$ position respectively. On the other hand $\mathrm{C} 4$ was placed by all users at last position.

- Q4: In which condition you sensed more the presence of your collaborator? Classify in order of preference.

(a) $\mathrm{C} 1$ (b) $\mathrm{C} 2$ (c) $\mathrm{C} 3$ (d) $\mathrm{C} 4$

Similarly for this also 90 percent subjects placed C3 at first priority while $10 \%$ put it on the 2nd. C2 got 10\%, $70 \%$ and $20 \%$ opinions for $1 \mathrm{st}$, 2 nd and 3 rd priority positions respectively. C1 was marked by $20 \%$ and $80 \%$ users for 2 nd and 3rd position respectively. On the other hand $\mathrm{C} 4$ was placed by all users at last position.

- Q5: What condition helped you more to establish coordination with your collaborator? Classify in order of preference.

(a) $\mathrm{C} 1$ (b) $\mathrm{C} 2$ (c) $\mathrm{C} 3$ (d) $\mathrm{C} 4$

For this question the priority percentage for conditions $(\mathrm{C} 1, \mathrm{C} 2$, $\mathrm{C} 3$, and $\mathrm{C} 4$ ) is exactly the same as for question no. 4 .

To summarize, we can say that C3 (shadow + arrows + oral communication) is the most preferable condition and users placed it on first priority position. In C3 condition users deduced cylinder's position with respect to the torus via shadow and status of the collaborator through arrows, while oral communication enhanced awareness and realism. Furthermore $\mathrm{C} 2, \mathrm{C} 1$ and $\mathrm{C} 4$ were placed on $2 \mathrm{nd}$, 3rd and $4^{\text {th }}$ position respectively.

\subsection{User learning}

In Fig. 12 the four trials (T1, T2, T3 and T4) are represented along $\mathrm{X}$-axis. Conditions are differentiated through different colors. The results show that applying condition $\mathrm{C} 1$, the subjects completed the task in $30.27 \mathrm{sec}(\mathrm{std}=3.25)$ in the first trial and in $27.2 \mathrm{sec}(\mathrm{std}=3.52)$ in the fourth trial. They completed the task under condition C2 in a mean time of 29.15 $\sec (\mathrm{std}=6.55)$ in the first trial, while took $23.6 \mathrm{sec}(\mathrm{std}=3.6)$ in the fourth trial.

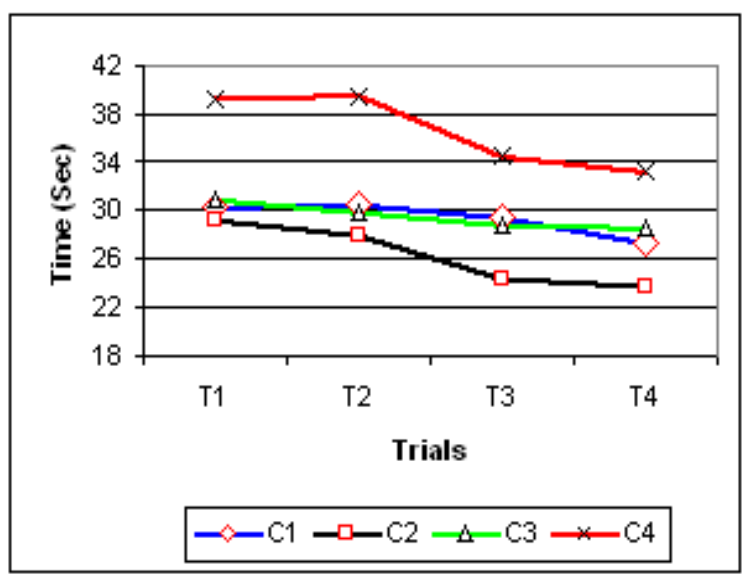

Fig. 12. Illustration of cooperative user learning in various conditions 
Similarly we have the mean time of $30.75 \mathrm{sec}(\mathrm{std}=4.05)$ under condition $\mathrm{C} 3$ for the first trial and $28.75 \mathrm{sec}(\mathrm{std}=3.52)$ for the last trial. We got mean time of $39.27 \mathrm{sec}$ ( $\mathrm{std}=5.51$ ) under condition $\mathrm{C} 4$ for the first trial and $33.15 \mathrm{sec}(\mathrm{std}=6.94)$ for the fourth trial (see figure). Therefore, we have performance improvement of 4.46, 19.03, 7.15 and 15.58 percent for conditions $\mathrm{C} 1, \mathrm{C} 2, \mathrm{C} 3$ and $\mathrm{C} 4$ respectively.

\section{COMPARATIVE ANALYSIS}

In this section we compare the task completion time for $\mathrm{C} 1$, $\mathrm{C} 2$ and $\mathrm{C} 4$ of the experiment I with those of the same conditions of the experiment II. The condition $\mathrm{C} 1$ has means of $30.07 \mathrm{sec}$ (std 6.17) and $6.34 \mathrm{sec}$ (std 1.5) for experiment II and I respectively and gives significant $(\mathrm{F}(2,9)=139.54, \mathrm{P}<0.05)$ ANOVA. The condition $\mathrm{C} 2$ has means of $28.38 \mathrm{sec}$ (std 3.11) and $6.26 \mathrm{sec}$ (std 0.82 ) for experiment II and I respectively and the ANOVA $(\mathrm{F}(2,9)=250.36, \mathrm{P}<0.05)$ result is also significant. Similarly for C4 we have means of $38.31 \mathrm{sec}$ (std 7.94) and $8.09 \mathrm{sec}$ (std 1.13) for experiment II and I respectively and the ANOVA $(\mathrm{F}(2,9)=141.83, \mathrm{P}<0.05)$ result is significant.

Similarly comparing the errors for C1 (8.6 errors, std 4.6), C2 (6.6 errors, std 3.5) and C4 (11.7 errors, std 5.7) of the experiments II (see Fig. 11) with the errors for the corresponding conditions $(\mathrm{C} 1=1.16$ errors ( $\operatorname{std} 0.72), \mathrm{C} 2=1.17$ errors (std 0.48) and $\mathrm{C} 4=1.47$ errors (std 1.02)) of experiment I (see Fig. 7) give significant differences. All these indicate that the task is easier to accomplish in single user setup as compared to the cooperative setup. It is also clear that shadow aids users in task accomplishment in both setups while the arrow enhances user performance in cooperative manipulation. The arrow is useful in cooperative manipulation because it not only gives feedback to the user about his end effector but also about the collaborator.

\section{CONCLUSION}

In this paper we simulated the use of two string based parallel robots in cooperative teleoperation task. Two users setting on separate machines connected through local area network operate each robot. In addition, the use of visual aids (i.e. shadows and arrows) and oral communication was investigated for their effects on cooperation, co-presence and users performance. Ten volunteer subjects cooperatively performed a peg-in-hole task. Another group of ten subjects performed the same task in a single user setup. Results revealed that shadow has a significant effect on task execution while arrows and oral communication not only increase users' performance but also enhance the sense of co-presence and awareness. We also observed that cooperative manipulation is more complex as compare to single user manipulation. Moreover we found that the addition of visual cues (arrows and shadows) and oral communication greatly helped users in cooperative manipulation of objects in the VE. Secondly these aids, specially arrows and oral communication also increased users' performance and enabled them to perceive each other's actions. Future work will be carried out to integrate the force feedback modality and examine its effects on cooperative task. Furthermore we will implement the system on long distance network (i.e. Internet) and investigate the influence of network delay.

\section{REFERENCES}

[1] www.teamspeak.com

[2] www.polhemus.com.

[3] M. O. Alhalabi and S. Horiguchi. Tele-handshake: A cooperative shared haptic virtual environment. in Proceedings of EuroHaptics, pages 60-64, 2001.

[4] J. Allard, S. Cotin, F. Faure, P.-J. Bensoussan, F. Poyer, C. Duriez, H. Delingette, and L. Grisoni. Sofa :an open source framework for medical simulation. In Medicine Meets Virtual Reality (MMVR), 2007.

[5] C. Basdogan, C. hao Ho, M. A. Srinivasan, and M. Slater. An experimental study on the role of touch in shared virtual environments. In ACM Transactions on Computer-Human Interaction, 2000.

[6] C. Basdogan, C.-H. Ho, M. A. Srinivasan, and M. Slater. Virtual training for a manual assembly task. In Haptics-e, volume 2, 2001.

[7] J. W. Chastine, J. C. Brooks, Y. Zhu, G. S. Owen, R. W. Harrison, and I. T. Weber. Ammp-vis: a collaborative virtual environment for molecular modeling. In VRST '05: Proceedings of the ACM symposium on Virtual reality software and technology, pages 8-15, New York, NY, USA, 2005. ACM.

[8] M. David, B. Arnaldi, and N. Plouzeau. A general framework for cooperative manipulation in virtual environments. Virtual Environments'99 Proceedings of the Eurographics Workshop, pages 169-178, 1999.

[9] C. Eckes, K. Biatov, F. Hlsken, J. Khler, P. Breuer, P. Branco, and L. Encarnacao. Towards sociable virtual humans: Multimodal recognition of human input and behavior. The International Journal of Virtual Reality, 6(4):21-30, 2007.

[10] P. M. Fitts. The information capacity of the human motor system in controlling the amplitude of movements. Journal of Experimental Psychology, pages 381-391, 1954

[11] D. M. Gaba. The future vision of simulation in health care. In Qual Saf Health Care, 2004.

[12] C. Greenhalgh and S. Benford. Massive: a collaborative virtual environment for teleconferencing. ACM Transactions on Computer Human Interaction, 2(3):239-261, 1995.

[13] I. Heldal, M. Spante, and M. Connell. Are two heads better than one?: object-focused work in physical and in virtual environments. VRST '06: Proceedings of the ACM symposium on Virtual reality software and technology, pages 287-296, 2006.

[14] R. J. Hubbold. Collaborative stretcher carrying: a case study. In Eighth Eurographics Workshop on Virtual Environments, 2002.

[15] J. Jordan, J. Mortensen, M. Oliveira, M. Slater, B. K. Tay, J. Kim, and M. A. Srinivasan. Collaboration in a mediated haptic environment. The 5th Annual International Workshop on Presence, 2002.

[16] N. Magnenat-Thalmann, P. Volino, U. Bonanni, I. Summers, M. Bergamasco, F. Salsedo, and F.-E. Wolter. From physics-based simulation to the touching of textiles: The haptex project. The International Journal of Virtual Reality, 6(3):35-44, 2007.

[17] M. Massimino and T. Sheridan. Variable force and visual feedback effects on teleoperator man/machine performance. In Proceedings of the NASA Conference on Space Telerobotic, pages 1751-1756, Pasadena, CA, Jan 1989.

[18] K. Montgomery, C. Bruyns, J. Brown, S. Sorkin, F. Mazzella, G. Thonier, A. Tellier, B. Lerman, and A. Menon. Spring: A general framework for collaborative, real-time surgical simulation. In Medicine Meets Virtual Reality (MMVR 2002), pages 23-26. IOS Press, 2002.

[19] J. Mortensen, V. Vinayagamoorthy, M. Slater, A. Steed, B. Lok, and M. C. Whitton. Collaboration in tele-immersive environments. In Proceedings of the workshop on Virtual environments, pages 93-101, 2002. 
[20] A. P. Olsson, C. R. Carignan, and J. Tang. Cooperative control of virtual objects using haptic teleoperation over the internet. Proceeding of $5^{\text {th }}$ Interantional Conference Disability Virtual Reality, 2004.

[21] O. Otto, D. Roberts, and R. Wolff. A review on effective closely-coupled collaboration using immersive cve's. In VRCIA '06: Proceedings of the 2006 ACM international conference on Virtual reality continuum and its applications, pages 145-154. ACM, 2006.

[22] G. V. Popescu, G. Burdea, and R. Boian. An experimental study on the role of touch in shared virtual environments. In ACM Transactions on Computer-Human Interaction, volume 7, 2000.

[23] P. Richard, G. Birebent, P. Coiffet, G. Burdea, D. Gomez, and N. Langrana. Effect of frame rate and force feedback on virtual object manipulation. PRESENCE : Massachusetts Institute of Technology, 5(1):95-108, 1996.

[24] P. Richard, D. Chamaret, F.-X. Inglese, P. Lucidarme, and J.-L. Ferrier. Human-scale virtual environment for product design: Effect of sensory substitution. International Journal of Virtual Reality, 5(2):3744, 2006.

[25] G. Riva, M. Bacchetta, G. Cesa, and E. Molinari. Cybertherapy: Internet and virtual reality as assessment and rehabilitation tools for clinical psychology and neuroscience. In IOS Press, pages 121-164, 2004.

[26] B. W. S. Marks, J. Windsor. Collaborative soft object manipulation for game engine-based virtual reality surgery simulators. Proceedings of Image and Vision Computing, pages 205-210, 2007.

[27] E.-L. Sallnas, K. Rassmus-Grohn, and C. Sjostrom. Supporting presence in collaborative environments by haptic force feedback. ACM Trans. Comput.-Hum. Interact., 7(4):461-476, 2000.

[28] M. Sato. Development of string-based force display: Spidar. In International Conference on Virtual Systems and Multi-Media (VSMM), 2002.

[29] X. Shen, F. Bogsanyi, L. Ni, and N. Georganas. A heterogeneous scalable architecture for collaborative haptics environments. Proceedings of the 2nd IEEE Internatioal Workshop on Haptic, Audio and Visual Environments and Their Applications, pages 113 -1187, 2003.

[30] S. Shirmohammadi and N. D. Georganas. An end-to-end communication architecture for collaborative virtual environments. Comput. Netw., 35(2-3):351-367, 2001.

[31] S. Ullah, P. Richard, S. Otmane, and M. Mallem. The effect of haptic guides on human performance in virtual environments. In Proceedings of 4th International Conference on Computer Graphics Theory and Applications, pages 322-327, 2009.

[32] B. Unger, A. Nicolaidis, P. Berkelman, A. Thompson, R. Klatzky, and R. Hollis. Comparison of $3 \mathrm{~d}$ haptic peg-in-hole tasks in real and virtual environments. In Proceedings of the 2001 IEEE/RSJ International Conference on Intelligent Robots and Systems (IROS '01), volume 3, pages 1751-1756, September 2001.

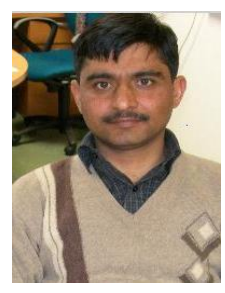

Sehat Ullah was born in 1977 in Pakistan. He got MSc degree in Computer science from university of Peshawar in 2001. He has been working as lecturer at the University of Malakand, Pakistan since 2003. He did his MS degree in Virtual Reality and intelligent systems from the University of Evry, France in 2007. Currently he is doing his $\mathrm{PhD}$ research in IBISC Laboratory University of Evry and LISA Laboratory University of Angers, France.

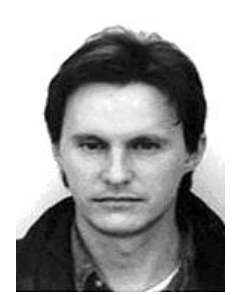

Paul Richard was born in Ngrepelisse, France, in 1964. $\mathrm{He}$ received his BS and MS in Electronics and Automation from the University of Nice Sophia-Antipolis in 1990 and 1991 respectively. He received a PhD in Robotics from the University of Paris VI in 1996. Dr. RICHARD is currently assistant Professor at the university of Angers - ISTIA (Institute des Sciences et Techniques de l'ingenieur d'Angers) and responsible for the virtual reality research activities of the Laboratoire d'ingnierie des systems automatiss d'Angers (LISA). For more than a decade his research has focused on virtual reality, multi-modal interaction and human performance in virtual environments. Dr. RICHARD was Co-Chair of VRIC (Virtual Reality International Conference) held in LAVAL in 2001, 2002 and 2003.

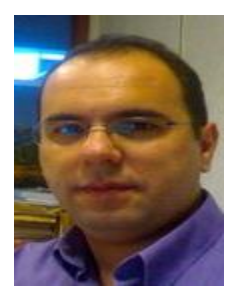

Samir Otmane received $\mathrm{PhD}$ degree in computer science from the University of Evry (France) in 2000. $\mathrm{He}$ is associate professor in computer science department of the University of Evry. He has been working on Virtual Reality and Augmented Reality applied to telerobotics (ARITI Project). He is working in the area of Virtual and Augmented Reality and CSCW (Computer Supported Collaborative Work). His current research efforts are focused on multimodal and collaborative 3D interaction in mixed reality environments.

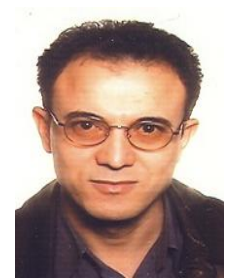

Malik Mallem received a $\mathrm{PhD}$ degree in robotics and computer sciences from University of Paris XII , in 1990. His research deals with Augmented Reality(AR) and Collaborative Work applied to robotics and telerobotics at IBISC- Complex System Laboratory CNRS FRE 3190, Evry, France. Since 1999, he is Professor at the University of Evry and head of AR team which is implied in several French and European projects.

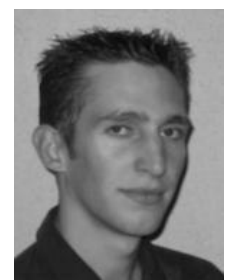

Mickaël Naud received his $\mathrm{PhD}$ in 2008 from the University of Angers. He is currently a researcher at Laboratoire d'ingénierie des systèmes automatisés d'Angers (LISA). He received a BS in computer science from the University of Angers in 2003 and a MS in virtual reality from the University of Maine, France in 2004. His research activities and interests are virtual reality, multi-modal interaction, and fashion design. 